\title{
Über die Behandlung des Trachoms
}

\author{
Von \\ Dr. K. Kiribuchi, \\ Tokio.
}

Die moderne praktische Medizin ist noch nicht so weit entwickelt, dass man irgendeinem Behandlungsverfahren des Trachoms unbedingt und ausschliesslich die Herrschaft zuerkennen kann. Unter verschiedenen Behandlungsmethoden gibt es zwar rein medikamentöse, mechanische, chirurgische, mechanisch-medikamentöse oder mechanisch-chirurgische, Galvanokaustik, Lichttherapie und Ionenbehandlung usw. Jede hat ihre Lichtund Schattenseite, so dass sie von der einen Seite angenommen, von der andern abgelehnt wird.

Seit langem hegten wir den Wunsch, nach einer langjährigen exakten Nachprüfung aller möglichen Methoden der Trachombehandlung an reichem Material, eine für alle Fälle von Trachom am besten indizierte Therapie zu ermitteln. Durch die Erfahrungen an meiner in Tokio von 1901 an ausgeübten Praxis komme ich zu folgenden Schlüssen:

a) Die medikamentöse Behandlung ist zwar, weil sie das Gewebe nicht lädiert, das beste Heilverfahren, aber da wir noch kein spezifisches Mittel gegen Trachom kennen, nimmt es Monate, ja sogar über Jahre in Anspruch, so dass die Patienten sich vorzeitig der Behandlung entziehen und dadurch ein voller Heilerfolg nicht erreicht werden kann. Von den Arzneimitteln möchte ich als die wirksamsten in erster Linie Jod-, Kupfer-, Quecksilberund Silberpräparate empfehlen.

b) Medikamentös-mechanische Behandlung wie Keiningsches Verfahren wirkt zwar palliativ, aber nicht radikal heilend, so dass es leicht zu Rezidiven kommt. Eine von Keining vorgeschlagene zu konzentrierte Sublimatlösung und zu heftige Ab- 
reibung hat eine noch stärkere Narbenbildung zur Folge, als das Leiden selbst.

c) Die viel vorgenommene Ausrollung und Ausbürsten (letzteres viel in Japan angewendet) hat relativ erfolgreiche Wirkung, aber bei dieser Methode dauert die Heilung zu lange.

d) Ausschneidung (von $\mathrm{Jak} \mathrm{obson-Heis} \mathrm{r}$ a th prinzipiell betont, von $\mathrm{Kuhnt}$ als "kombinierte Exzision" bezeichnete Methode) verkürzt bedeutend die Heilungsdauer und verhütet sekundäre Hornhauterkrankungen oder hat sichere Heilwirkung bei letzteren. Aber die ausgeschnittene Conjunctiva kann sich einerseits, trotz Infiltration, Verdickung und sogar leichter Narbenveränderung, später zum grossen Teil regenerieren. Anderseits kann der infiltrierte und verdickte Tarsus, ein Schutzorgan des Auges, bei intakten oder veränderten Meibomschen Drüsen ohne Exzision zur Heilung gebracht werden. Es ist deshalb wünschenswert, wenn möglich, eine eingreifende Operation zu vermeiden.

e) Die von einigen emplohlenen Radiumstrahlen sind nicht so erfolgreich, während die ultravioletten Strahlen zwar intensiv wirken, aber aus äusseren Umständen nicht allgemein anwendbar sind.

Die Hauptpunkte der Trachombehandlung sind folgende:

1. In erster Linie ist es wünschenswert, die Heilungsdauer möglichst abzukürzen und die Gewebsopferung, wenn möglich, $\mathrm{zu}$ vermeiden.

2. Viele Kranke haben wegen der Gewöhnung an das Leiden Abneigung gegen eine Operation, solange quälende Komplikationen nicht eingetreten sind. Es muss die allgemein anwendbare Operation für die Trachomleidenden eine möglichst einfache - wenigstens dem Kranken einfach erscheinende - und ohne Verschwendung der Zeit (in durchseuchten Gegendenl) ausführbare Methode sein.

3. Die Trachombehandlung möchte eine für alle Fälle ausführbare sein. Es würde zurzeit, wo es weder ein Spezifikum gegen Trachom noch eine als rationell allgemein anerkannte mechanische oder chirurgische Therapie gibt, nötig sein, die Behandlung in kombinierten Methoden zu suchen.

4. Bekanntlich zeigt der Tarsus häufig schon im Frühstadium des Trachoms in seinen oberen Randpartien Infiltration, Hyperämie und Verdickung. In jedem Falle ist daran festzuhalten, dass die 
starke Mitbeteiligung des Tarsus eine ernste Komplikation darstellt. Sie widersteht hartnäckig den therapeutischen Bemühungen, und verursacht die üblen Rezidive des Trachoms, so dass die Heilung sich über Jahre erstreckt. Dass die pannöse Erkrankung häufig die Cornea von oben her zu überziehen beginnt, ist eine bekannte Tatsache. Bevor der Pannus zum Ausbruch kommt, sieht man eine in dem oberen Abschnitt der Augapfelbindehaut auftretende subkonjunktivale Gefässinjektion, die bald ihren Höhepunkt an der Grenze der Hornhaut erreichen kann und dann das obere Segment der Hornhaut umgibt. Bald lässt sich auch hier eine deutliche Schwellung des Limbus erkennen. Sehr bald darauf breitet die Vaskularisation und Infiltration sich über die Cornea aus.

Bei der mikroskopischen Untersuchung der Pannusentwicklung sieht man zuerst in der Übergangsfalte bzw. im Supratarsalgewebe, ja sogar in der oberen Partie des Tarsus eine trachomatöse Veränderung entstehen. Mit diesem Herde steht die oben erwähnte Erweiterung der Subkonjunktivalgefässe der Conjunctiva bulbi in Verbindung, in deren Umgebung reichliche Zellenhaufen anzutreffen sind. Diese Subkonjunktivalgefässe ziehen vom oberen Teil der Augapfelbindehaut nach der Cornea, sie erreichen, begleitet von einer charakteristischen oberflächlichen subepithelialen Zellinfiltration, den Limbus corneae. Hier entsteht sehr bald eine hochgradige Zellinfiltration und Vaskularisation, während jene oberflächliche subepitheliale Zellinfiltration später oder fast gleichzeitig mit der Limbusinfiltration in Verbindung tritt. Vom Limbusherde aus gehen die Gefässe und Zellinfiltrationen auf die Cornea über, und so entsteht der Pannus. Der Tarsus des Oberlides ist im Vergleich mit dem des Unterlides bedeutend grösser und gefässreicher, besonders ist der obere Teil des Tarsus und das Supratarsalgewebe am gefässreichsten, ferner sind Plateau und Rinnen (nach Hans Vir chow) oben beträchtlicher, dagegen unten, in halber Höhe des Tarsus, nur in Spuren entwickelt. Dass dadurch die obere Partie der Tarsalgegend und Übergangsfalte einen günstigeren Boden für Trachomerreger bildet und infolgedessen hier das Trachom bis in das gefässreiche Tarsusgewebe vordringen und hier der Heilung hartnäckigen Widerstand leisten kann, ist leicht verständlich. Auch die häufige Entwicklung des Pannus am oberen Hornhautabschnitt scheint sich aus dem oben erwähnten anatomischen Verhalten zu erklären. Daher sind die 
obere Tarsuspartie und die Übergangsfalte des oberen Lides eine wichtige Angriffsgegend beim Heilungsverfahren.

5. Allgemeiner körperlicher Zustand des Kranken.

Bei den Trachomleidenden mit gesundem, kräftigem Körperbau scheint es, als ob die natürliche Heilungskraft dem Krankheitsprozesse entgegenwirke und die Heilung befördere. Bei solchen Leuten ist das Leiden viel schneller und grändlicher heilbar. Dagegen scheint es bei Individuen mit lymphatischer Diathese, bei Skrophulösen, Tuberkulösen, Nephritikern, Diabetikern usw., und besonders bei Luetikern, als ob das Trachom häufig hartnäckiger auftritt und leichter rezidiviert. Es ergibt sich daraus, dass es unerlässlich ist, bei der Trachomtherapie den allgemeinen Körperzustand des Patienten zu berücksichtigen und eine passende Behandlung einzuleiten.

6. Wie bekannt ist, darf man bei der Trachomtherapie nicht versäumen, phimotische Verengerungen der Lidspalte, Stellungsanomalien der Lider, insbesondere der Lidränder, Erkrankungen des tränenableitenden Apparates zu beseitigen.

In meiner Klinik wird das Trachom in folgender Weise behandelt:

Nach Einträufelung einer 4\%igen Kokainlösung (bei empfindlichen Personen wird eine $0,5 \%$ ige Kokainlösung subkonjunktival injiziert, noch stärkere Lösung wird vermieden, da dieselbe auf das Gewebe schädlich wirkt und die Resorption der Infiltration verhindert) wird das obere Lid des Patienten, meist in sitzender Lage im Poliklinikzimmer, umgestülpt, dann wird mit dem linken Daumen (oder Zeige- und Mittelfinger) durch Vermittelung des Sublimatwattebäuschchens der Lidrand (von der Konjunktivalseite aus) nach dem oberen Orbitalrande gehoben und leicht gedrückt, während mit dem rechten Finger durch das untere Lid ein Druck auf den Augapfel ausgeübt wird. Der Patient blickt der Weisung gemäss stark nach unten, dann tritt die Übergangsfalte leicht in ihrer Totalität hervor, auch ist die ganze obere Conjunctiva bulbi faltenlos ausgebreitet. Gleichzeitig wird man in der oberen Tarsalgegend mit Bindehautveränderungen zusammen der tiefliegenden Verdickung und Infiltration des Tarsus gewahr.

Als zweiter Akt werden in der Utbergangsfalte mit einem mit der rechten Hand gehaltenen schmalen zweischneidigen, leicht konkav gebogenen Instrumente (Stichler) in die Granula und In- 
filtration zahllose Einstiche gemacht. Ebenso zahlreiche Einstiche macht man am oberen Teile des Tarsus senkrecht auf seine Oberfläche tiefer, bis man in das Tarsusgewebe gelangt. Es ist nötig, besonders am konvexen Rand des Tarsus, mehrere Einstiche vorzunehmen (eine solche Knorpeleinstichelung wurde auch von $K u h n t$ vorgenommen). Da während der ganzen Zeit eine profuse Blutung vorkommt, so muss die Bindehautoberfläche mit einem in eine antiseptische Lösung getauchten und ausgedrückten Wattebäuschchen abgewischt werden, damit das Operationsgebiet gut sichtbar bleibt.

Falls die Veränderungen der Tarsalbindehaut ausgebreitete sind oder schon bis zum Lidrand reichen, sticht man hauptsächlich die Granula und macht nebenbei mehr oder weniger Einstiche in die Infiltrationsstelle bis in den Tarsus, dieselben brauchen aber nicht so zahlreich zu sein.

Dritter Akt: Mit einem mit 0,2\% Sublimatlösung durchtränkten Wattebäuschchen massiert man die Granula und die Infiltration der Übergangsfalte in der Richtung von der Conjunctiva bulbi nach dem Tarsus zu drückend. Dann senkt man den den linken Lidrand fixierenden Finger ein wenig nach unten, drückt den ganzen oberen Tarsusteil zwischen diesem Finger und Sublimatwatte (die hinter dem Tarsusrande eingeschoben wurde) aus und reibt noch ein- bis zweimal die Oberfläche dieser Gegend mit Sublimatwatte ab. Alsdann werden die Granula und Infiltration der Übergangsfalte und oberen Tarsusgegend herausgedrückt und gereinigt, der obere Tarsusteil wird weich. Endlich reibt man die andere Partie der Conjunctiva tarsi ab.

Vierter Akt: Dann folgt das Abspülen und Reinigen der Wundflächen der Conjunktiva. Dabei ist eine immer vorkommende reichliche Blutung wünschenswert. Darauf streicht man mittels trockener sterilisierter Watte Airolpulver auf die ganze Wundfläche der Conjunktiva. Falls ein Pannusgeschwür vorhanden ist, dann ist auch Airolbetupfung notwendig.

Es bedarf unter einer Assistenz zur Ausführung der ganzen Operation an den oberen und unteren Lidern der beiden Augen nur einer Zeit von ungefähr 3 Minuten. Darauf lässt man den Patienten etwa $1 / 2$ Stunde lang kalte Umschläge machen. Wenn man zuletzt die Konjunktivalfläche von den mit dem Sekret vermengten Airolmassen reinigt, ist der Patient imstande, mit offenen Augen zu gehen. Gewöhnlich führe ich die genannte Operation 
neben dem auf dem Stuhl sitzenden Kranken, an dessen rechter Seite stehend, aus. Selten operiere ich den empfindlichen $\mathrm{Pa}-$ tienten auf dem Operationstisch in liegender Lage. Alle werden ambulatorisch behandelt.

Einen Tag nach der Operation klagen die Operierten nicht mehr über Schmerzen oder Unbehagen, sondern die meisten fühlen sich an den Augen erleichtert. Vom dritten bis vierten Tage an können die Operierten ihrer Beschäftigung nachgehen.

Nachbehandlung: An den ersten drei Tagen ist der Konjunktivalsack auszuspülen und zu reinigen, nachher ist Airolpulver auf die Wundfläche zu streichen (fein gepulvertes Airol muss in dünner Schicht gestrichen werden, da sonst quälendes Fremdkörpergefühl entsteht). Vom vierten Tage an reibt man täglich (oder alle zwei Tage) einmal leicht mit einem $0,2 \%$ sublimatgetränkten Wattebäuschchen den oberen Tarsalteil und leichter die Übergangsfalte ab. Darauf wird der Konjunktivalsack abgespült, gereinigt, dann Airolpulver auf die Wundfläche der oberen Tarsalgegend und Übergangsfalte gestrichen. Die umgestülpten Lider werden untereinander geschoben, und man reibt durch kreisförmige Bewegungen die Konjunktivalflächen aneinander. So bleibt das auf den oberen Tarsus und die Übergangsfalte gestrichene Airolpulver zum Teil zurück, und das übrige Airol wird auf die ganze Konjunktivalfläche in sehr dünner Schicht verteilt. Ferner befördert die durch das Aneinanderreiben der Conjunctivae hervorgerufene Hyperämie die Resorption der zelligen Infiltration mehr oder weniger.

Der erste, welcher Airol für die Trachomtherapie anwandte, war Tausig (1896). Obgleich nachher die Airolanwendung gegen Trachom von einigen angenommen wurde, wurde im allgemeinen das Mittel ausser acht gelassen. Seit über 10 Jahren habe ich seine Wirkung gegen Trachom klinisch geprüft. Das Airol ist in der Tat nach meiner Erfahrung unter den antitrachomatösen Mitteln das empfehlenswerteste. Es zersetzt sich im Konjunktivalsack in seine Komponenten. Das naszierende Jod wirkt tief in das Gewebe. Tannin und Wismut üben auch wohl ihre günstigen Wirkungen an der Oberfläche der Conjunktiva aus.

Unsere Trachomtherapie ist, wie oben erwähnt, eine chirurgisch-mechanisch-medikamentöse. Sie findet ihre Anwendung in allen Fällen des Trachoms, ohne Kontraindikationen. Auch im Stadium cicatriceum, wo die Conjunktiva in Narbenschrumpfung 
übergeht und ausheilt, während doch die pannöse Erkrankung der Cornea besteht, ist unsere Behandlung indiziert, ferner im Falle des akuten Trachoms, das in seinem Verlaufe durch Sichtbarwerden der Granula und Auftreten der anderen Symptome als solches diagnostierbar wird. In diesen Fällen sind die Stichelungen des Tarsus selten nötig, es genügen meist nur die der Conjunktiva.

Bei Kindern verläuft das Trachom meistens leicht, so dass die Stichelungen bei kleinen Kindern meist nicht nötig, nur bei grösseren Kindern die Stichelungen der Conjunktiva und sehr selten auch die des Tarsus vorgenommen werden müssen.

Dass unsere Behandlungsmethode immer einen überraschenden Erfolg zeigt, lässt sich durch folgende Tatsache beweisen: Es waren viele Patienten, welche über 10 Jahre, ja sogar über 20 Jahre lang an hartnäckigem Trachom litten und trotz langer ärztlicher Behandlungen in allen Gegenden von ihren üblen Leiden nicht befreit wurden. Sie brachten unsere erfolgreiche Behandlung in Erfahrung, worauf sie gleich unsere Poliklinik besuchten. Der Heilerfolg trat durch unsere Behandlungen bei allen bald ein.

Auf Grund meiner reichen Erfahrungen an über 6000 Augen darf ich wohl folgendes behaupten:

1. Die Methode verkürzt ganz beträchtlich den Heilungsverlauf; derselbe weicht kaum von dem bei der kombinierten Exzision ab. Die Schädigung der Bindehaut ist geringer wie die durch Ausrollen oder Ausbürsten. Die zahlreichen Stichelungen des oberen Teils des Tarsus bringen im späteren Verlaufe keinen Nachteil für das Auge.

2. Sekundäre Hornhauterkrankungen werden am sichersten und schnell zur Heilung gebracht; ihr Auftreten kann auch wohl verhütet werden.

3. Wiederaufflackern der Entzündung, Rezidive bzw. Reinfektion können vielleicht ganz hintangehalten werden, wenn die Nachbehandlung sorgfältig durchgeführt wird.

Nach lege artis ausgeführtem Verfahren konnte ich die gründliche Heilung im günstigsten Falle nach 4 Wochen, spätestens nach 3-4 Monaten erzielen. Im Durchschnitt, wenn die Affektion nicht $\mathrm{zu}$ schwer und hartnäckig ist, konnte ich die Heilung nach etwa anderthalb Monaten feststellen. Bei schweren und ziemlich hartnäckigen Fällen war es selten nötig, etwa nach eịnem 
Monat die Operation zu wiederholen, was sich die Kranken in der Regel, da sie schon den Operationserfolg im voraus wissen, gern gefallen lassen.

Besonders günstig werden durch die Operation die sekundären Hornhautaffektionen beeinflusst. Am Tage nach der Operation bessert sich schon der Pannus und die Ulcera. Der Pannus wird Tag für Tag durchsichtiger und kleiner, das Sehvermögen stellt sich bald wieder her. (Sehr selten ist, bei nicht ganz sorgfältiger Durchführung der Operátion, am nächsten Tage das pannöse Geschwür noch nicht gereinigt. Man macht dann die Stichelungen an dem betreffenden Tarsusrand und in der Übergangsfalte und pudert Airolpulver auf. Es tritt dann bald Heilung ein.)

Die oben beschriebene Behandlungsmethode steht in ihrer Wirkung nicht nur der kombinierten Exzision nicht nach, sondern verschont das Gewebe. Sie ist wirksamer als das Ausrollen oder Ausbürsten, da sie die für die Trachomtherapie wichtigste Partie - den oberen Teil des Tarsus selbst - mit der Supratarsalgegend energisch angreift. Die Methode ist einfach und in allen Fällen anwendbar.

$\mathrm{Da}$ in relativ kurzer Zeit gründliche Heilung erzielt werden kann, bleiben die meisten Patienten geduldig und entziehen sich nicht der Behandlung vorzeitig.

Es würde mich freuen, wenn das von mir geübte Verfahren von anderer. Seite nachgeprüft werden würde. 